\title{
Synchronization of Sensory Gamma Oscillations Promotes Multisensory Communication
}

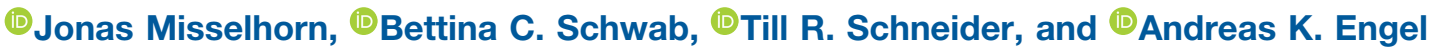

https://doi.org/10.1523/ENEURO.0101-19.2019

Department of Neurophysiology and Pathophysiology, University Medical Center Hamburg-Eppendorf, 20246 Hamburg, Germany

\begin{abstract}
Rhythmic neuronal activity in the gamma range is a signature of cortical processing and its synchronization across distant sites has been proposed as a fundamental mechanism of network interactions. While this has been shown within sensory streams, we tested whether cross talk between the senses relies on similar mechanisms. Direct sensory interactions in humans (male and female) were studied with a visual-tactile amplitude matching paradigm. In this task, congruent stimuli are associated with behavioral benefits, which are proposed to be mediated by increased binding between sensory cortices through coherent gamma oscillations. We tested this hypothesis by applying 4-in-1 multi-electrode transcranial alternating current stimulation (tACS) with $40 \mathrm{~Hz}$ over visual and somatosensory cortices. In phase stimulation $\left(0^{\circ}\right)$ was expected to strengthen binding and thereby enhance the congruence effect, while anti-phase $\left(180^{\circ}\right)$ stimulation was expected to have opposite effects. Gamma tACS was controlled by alpha $(10 \mathrm{~Hz})$ and sham stimulation, as well as by applying tACS unilaterally while visual-tactile stimuli were presented lateralized. Contrary to our expectations, gamma tACS over the relevant hemisphere delayed responses to congruent trials. Additionally, reanalysis of EEG data revealed decoupling of sensory gamma oscillations during congruent trials. We propose that gamma tACS prevented sensory decoupling and thereby limited the congruence effect. Together, our results favor the perspective that processing multisensory congruence involves corticocortical communication rather than feature binding. Furthermore, we found control stimulation over the irrelevant hemisphere to speed responses under alpha stimulation and to delay responses under gamma stimulation, consistent with the idea that contralateral alpha/gamma dynamics regulate cortical excitability.
\end{abstract}

Key words: coherence; communication; EEG; gamma; networks; tACS

\section{Significance Statement}

Cortical gamma oscillations structure segregated neural activity and were suggested to represent a fundamental mechanism of network communication. While there is ample evidence for the role of longrange gamma synchronization in unisensory processing, its significance in multisensory networks is still unclear. We show that coordinated sensory gamma oscillations play an important role for direct crossmodal interactions and propose that phase synchronization promotes communication between sensory cortices. To that end, we conducted a state-of-the-art multi-electrode transcranial alternating current stimulation experiment designed to modulate coherence between sensory cortices and analyzed connectivity in a previously recorded high-density EEG dataset. By complementing an interventional with an observational method, we provide novel evidence for the role of synchronized gamma oscillations in multisensory communication. 


\section{Introduction}

Perceiving the world through distinct sensory channels provides complementary as well as redundant and conflicting information about the environment. To structure these sensory signals, fundamental neuronal computations are concerned with cross-modal matching of sensory signals. On the neuronal and behavioral levels, processing cross-modally congruent stimuli is associated with enhanced efficiency when compared with incongruent or unimodal processing and often coincides with enhanced cortical activity (Ghazanfar and Schroeder, 2006) and behavioral benefits (Spence, 2011). Within sensory systems, such integrative processes likely involve corticocortical synchronization of high-frequency oscillatory activity (Engel et al., 2001; Fries, 2009). For instance, perceptual grouping and feature binding across cortical columns and hemispheric homologs of visual cortex have been shown to involve phase coupling of neuronal gamma band oscillations (Gray et al., 1989; Engel et al., 1991). Relatedly, it was suggested that synchronized oscillations might provide a solution to the binding problem (TallonBaudry et al., 1996; Treisman, 1996). Moreover, gamma oscillations have been proposed to constitute a framework that allows transmitting of coherent patterns of neural activity along sensory streams (Fries, 2015). Together, the coordination of gamma oscillations may enable structuring as well as transmitting sensory information within sensory networks and thereby likely plays an important role in orchestrating multisensory interactions (Keil and Senkowski, 2018).

A number of studies have investigated gamma band activity during multisensory perception. Visual stimulus detection, for instance, was shown to be improved by redundant auditory stimuli while gamma band responses in frontal cortex were enhanced (Senkowski et al., 2005, 2007). Recognition and classification of visual objects was improved by congruent auditory input showing increased gamma band power in temporal or parietal cortices (Yuval-Greenberg and Deouell, 2007; Schneider et al., 2008a). While the aforementioned studies showed multisensory modulations of gamma band power in association cortices, other studies also noted changes in sensory cortices (Krebber et al., 2015; Friese et al., 2016). For

Received March 15, 2019; accepted September 16, 2019; First published October 10, 2019.

The authors declare no competing financial interests.

Author contributions: J.M., B.C.S., T.R.S., and A.K.E. designed research; J.M. and B.C.S. performed research; J.M. analyzed data; J.M., B.C.S., T.R.S., and A.K.E. wrote the paper.

This research was supported by Deutsche Forschungsgemeinschaft Grants SFB 936/A3/Z1, SFB TRR 169/B1, SPP 1665/EN 533/13-1, and SPP 1665/ SCHN 1511/1-2; European Research Council (ERC) Grant ERC-2010-AdG269716.

Acknowledgments: We thank Karin Deazle for help with recruitment of participants and data recording.

Correspondence should be addressed to Jonas Misselhorn at j.misselhorn@uke.de.

https://doi.org/10.1523/ENEURO.0101-19.2019

Copyright (C) 2019 Misselhorn et al.

This is an open-access article distributed under the terms of the Creative Commons Attribution 4.0 International license, which permits unrestricted use, distribution and reproduction in any medium provided that the original work is properly attributed. instance, attention for suprathreshold audio-visual stimuli was associated with enhanced sensory gamma oscillations in both the visual and auditory cortices (Friese et al., 2016) and matching congruent visual-tactile motion stimuli induced enhanced gamma power in visual and somatosensory cortices (Krebber et al., 2015). Additionally, there is evidence for altered gamma oscillations underlying schizophrenia (Uhlhaas and Singer, 2010; Mulert et al., 2011; Curic et al., 2019). In these patients, aberrant multisensory integration was shown to be accompanied by altered gamma band dynamics in response to multisensory stimuli (Stone et al., 2014; Balz et al., 2016). Together, cross-modally corresponding or congruent stimuli typically induce strong local synchronization of gamma band oscillations in both sensory and association cortices.

In addition to local changes in gamma band activity, it was suggested that cross-modal interactions involve interareal phase synchronization of sensory gamma oscillations (Senkowski et al., 2008; Keil and Senkowski, 2018). Specifically, enhanced processing of crossmodally congruent stimuli might imply feature binding across modalities mediated by synchronization of sensory gamma oscillations. A constraint in testing this hypothesis is that differences in power constitute a bias for the computation of phase coherence (Bastos and Schoffelen, 2016). As reviewed above, many multisensory paradigms would, thus, not be suited for testing this prediction. Here, we used a paradigm that has not revealed differences in gamma power in sensory cortices during cross-modal matching (Misselhorn et al., 2019). In this task, participants match concurrent amplitude changes of visual and tactile stimuli that are either congruent (both increase or decrease in intensity) or incongruent (increase in one and decrease in the other modality). Following Senkowski et al. (2008), we assumed that congruence enhancement would entail increased coupling between sensory gamma oscillations. Modulating coupling between visual and somatosensory cortices should therefore influence the effect of cross-modal congruence. To test this hypothesis, we used focal multi-electrode transcranial alternating current stimulation (tACS) to modulate the synchrony of sensory gamma oscillations between visual and somatosensory cortices. Sham-controlled tACS was applied at either $10 \mathrm{~Hz}$ (alpha) or $40 \mathrm{~Hz}$ (gamma) with $0^{\circ}$ (in-phase) or $180^{\circ}$ (anti-phase) phase shift between montages. Additionally, we controlled the effect of stimulation by presenting lateralized stimuli and applying tACS unilaterally. We hypothesized task-specific effects of tACS only to occur when electrical and sensory stimulation were targeted at the same hemisphere, but no or only unspecific effects when electrical and sensory stimulation were targeted at different hemispheres. Specifically, we expected in-phase gamma stimulation over the relevant hemisphere to enhance the congruence effect by (1) speeding responses to congruent stimuli due to enhanced feature binding and (2) delaying responses to incongruent stimuli by imposing "false" feature binding. Gamma anti-phase tACS over the relevant hemisphere should show inverse effects. Additionally, we reanalyzed the aforementioned EEG data (Misselhorn et al., 2019) with respect to the coherence of 
A

\section{A \\ visual

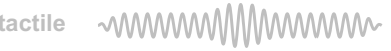

B

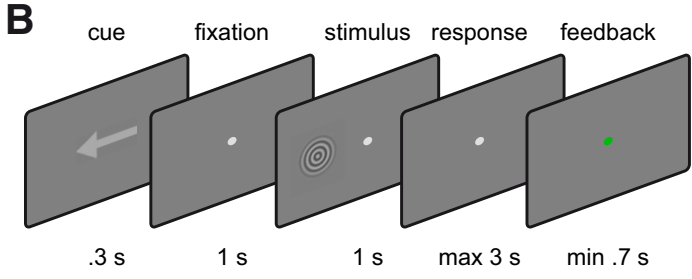

D

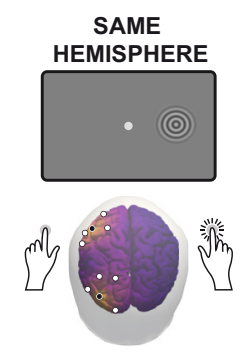

$1 \mathrm{~s}$

$\max 3 \mathrm{~s} \quad \min .7 \mathrm{~s}$

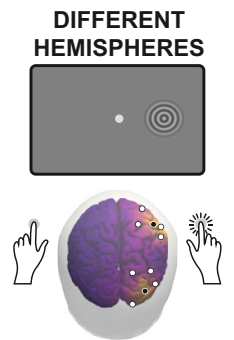

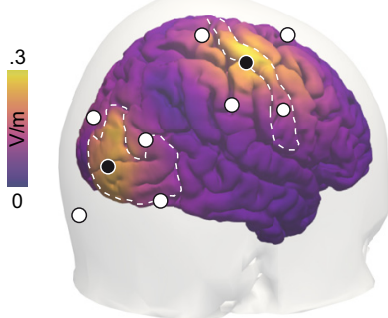

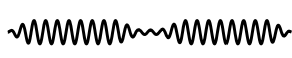

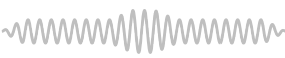

C

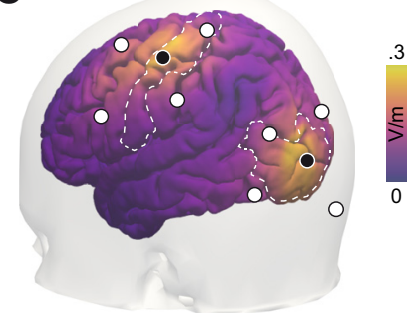

Figure 1. Experimental design. $\boldsymbol{A}$, visual-tactile stimuli were presented on each trial. Brief changes in stimulus intensity occurred concurrently in both modalities, either in the same direction ("congruent") or in different directions ("incongruent"). B, Each trial started with a central arrow that cued the left or right side reliably. After $1 \mathrm{~s}$ of central fixation, the visual-tactile stimulus was presented on the cued side. Participants were asked to maintain central fixation and report congruence of the presented stimulus. After response, participants received feedback. C, Multi-electrode tACS montage (black and white electrodes represent different polarities) and estimation of current density on cortical surface. Participants received either left or right hemispheric stimulation with two 4-in-1 montages over visual and somatosensory cortices. Color coding on cortical surface corresponds to the simulated maximum absolute field strength in V/m. $\boldsymbol{D}$, On a given trial, electrical and sensory stimulation could be targeted at the same hemisphere (left) or at different hemispheres (right).

gamma oscillations to inform the interpretation of the behavioral results from this study.

\section{Materials and Methods}

\section{tACS experiment \\ Participants}

Twenty-four participants were recruited and completed a training session, after which four participants dropped out due to insufficient performance ( $<60 \%$ accuracy). Twenty participants completed three experimental sessions (13 females; age, $25.3 \pm 4.5$ years). None of them had a history of neurologic or psychiatric disorders. All participants gave informed written consent and received monetary compensation for their participation. The local ethics committee approved the study, which was conducted in accordance with the Declaration of Helsinki.

\section{Experimental design}

Participants performed a spatially cued cross-modal amplitude matching task on visual-tactile stimuli. We presented a circular, expanding grating (diameter, $5^{\circ}$ visual angle) on a CRT screen (refresh rate, $120 \mathrm{~Hz}$; model HM204DTA, liyama) against a gray background as visual stimulation. Gratings were presented with $5^{\circ}$ visual angle offset to the left or right of the vertical meridian. Tactile stimulation was realized by a high-frequency vibration delivered to the fingertips of both index fingers $(250 \mathrm{~Hz}$ on C-2 tactors, Engineering Acoustics). Throughout the whole experiment participants kept fixation on a central fixation point. To induce a covert shift of attention, a centrally presented arrow cued the left or right side $(100 \%$ reliability, $300 \mathrm{~ms}$; Fig. 1B). After $1 \mathrm{~s}$, a visual-tactile stimulus was presented on the cued side only. That is, visual stimuli were presented to the left or right side of the fixation, and the tactile stimulus was presented to the left or right index finger. On each trial, both visual and tactile components underwent a brief suprathreshold change in intensity, either an increase or a decrease (Fig. 1A). Magnitudes of change were derived from a previous behavioral study using a similar paradigm (Misselhorn et al., 2016). Participants were asked to compare the change direction between visual and tactile components and report whether they changed congruently or incongruently (Fig. 1A). Responses were instructed to be given as fast as possible by using a foot switch. After a training session, participants completed two identical experimental sessions containing three blocks holding 192 trials. Experimental session used either alpha $(10 \mathrm{~Hz})$ or gamma (40 $\mathrm{Hz}$ ) stimulation. These canonical stimulation frequencies were chosen because previous studies showed behavioral as well as neurophysiological effects for these frequencies (Helfrich et al., 2014; Schwab et al., 2019). The order of experimental sessions was counterbalanced across participants. Experimental blocks featured inphase, anti-phase, or sham stimulation (for details, see Electrical stimulation). The order of stimulation conditions was counterbalanced across participants.

\section{Electrical stimulation}

Alternating currents were administered in 4-in-1 montages with current flow between the four outer electrodes and one central electrode (Patel et al., 2009; Saturnino et al., 2015) using $\mathrm{Ag} / \mathrm{AgCl}$ ring electrodes (diameter, 12 $\mathrm{mm}$ ). This configuration results in focal electric fields with peaks underneath the central electrode (Fig. 1C). For each participant, we prepared two of these montages designed to target primary visual and primary somatosensory cortices of one hemisphere, respectively. The side of 
stimulation was counterbalanced across participants. In conjunction with the lateralized experimental design, this resulted in equal proportions of trials in which electrical and sensory stimulation were targeted at the same hemisphere (Fig. 1D, left) or at different hemispheres (Fig. 1D, right). Before experimental blocks, stimulation was ramped up to $2 \mathrm{~mA}$ peak to peak within $10 \mathrm{~s}$. Sham blocks started with the same ramps but included no stimulation thereafter. For in-phase stimulation, we used the same waveforms for both montages. For anti-phase stimulation, one waveform was shifted by $180^{\circ}$. Two separate DC stimulators were used (DC-Stimulator Plus, Neuroconn). Stimulators were operated in external mode, allowing control of the current output via voltage input. The voltage signal was computed in Matlab and produced by a $\mathrm{Nl}$ DAQ device run with Labview (NI USB 6343, National Instruments). Impedances of each of the four outer electrodes relative to the central electrode were kept comparable within montages $(10-100 \mathrm{k} \Omega$ ). This is crucial because identical impedances were assumed for the simulation of electric fields.

\section{Simulation of electric fields}

Electrode positions for the 4-in-1 montages were chosen such that electric field strength was maximized in visual and somatosensory areas. Simulations of current flow were performed based on the lead field matrix $L$, which was computed for a realistic three-shell head model (Nolte and Dassios, 2005), and a cortical grid in MNI space, obtained by downsampling the Freesurfer template to 10,000 grid points (Desikan et al., 2006). The electric field at location $x$ was estimated by linear weighting of the lead field matrix $L$ with the injected currents $\alpha_{i}$, where $i$ denotes indices of the 10 stimulation electrodes, as follows:

$$
\vec{E}(\vec{x})=\sum_{i}\left(\overrightarrow{L(\vec{x})} \alpha_{i}\right) .
$$

Within visual and somatosensory regions, peak values of $0.3 \mathrm{~V} / \mathrm{m}$ were reached using currents with peak values of $1 \mathrm{~mA}(2 \mathrm{~mA}$ peak to peak). Focality was high as field strengths rapidly decreased when moving away from the central electrode (Fig. 1C). This ensured that effective electrical stimulation was confined to the targeted regions of one hemisphere only.

\section{Statistical analysis}

The effects of tACS were evaluated by analyzing accuracy and response times (RTs). First, we computed a repeated measures ANOVA with factors HEMISPHERE (same/different), FREQUENCY (alpha/gamma), STIMULATION (sham/in-phase/anti-phase), and CONGRUENCE (congruent/incongruent). Where necessary, the Greenhouse-Geisser correction was applied. Tables containing complete results from ANOVA are provided in Extended Data Table 1-1. Higher-order interactions were followed up by computing reduced ANOVA models and, finally, significant two-way interactions were followed up by a nonparametric analysis based on comparing RT distributions. To that end, we estimated cumulative distribution functions (CDFs) of RT distributions using a Gaussian kernel estimator (Botev et al., 2010). CDFs were estimated for RTs between 0 and $4 \mathrm{~s}$ using 1024 bins for each subcondition and participant. Next, we computed differences between CDFs and averaged across participants. To decide about the statistical significance of differences between CDFs, we constructed confidence intervals (Cls) by permutation tests. That is, we shuffled all data from a given interaction into two sets, computed CDFs and stored the difference between the CDFs of the two sets as the null-distribution (100,000 permutations). Two-sided Cls were constructed by finding percentiles (lower bound, $\alpha / 2$; upper bound, $100-\alpha / 2$ ) in the null distribution that reflect the range of positive and negative differences along the RT range that can be expected to result from random fluctuations. The final $\mathrm{Cls}$ are corrected for both (1) multiple testing due to condition contrasts and (2) multiple tests along the $\mathrm{RT}$ range, with an initial probability of false positives set to $\alpha=5 \%$. The latter source of multiple tests is especially critical because testing a range of values compared with testing one value (e.g., a central tendency as in ANOVA) profoundly inflates the probability of false positives. The first issue was dealt with by Bonferroni correction and yields $\alpha$. The second issue, however, would not be adequately dealt with by Bonferroni correction because the number of tests along the RT range is an arbitrary choice. Thus, instead of applying the $\alpha$ at each RT bin separately, we applied it globally to all RT bins collectively. That is, we counted instances of the null distribution (one instance is the null result from a single permutation) that fall outside the confidence interval at any RT bin. The global $\alpha$ was found by iteratively decreasing $\alpha$ until only the $\alpha$ percentage of all null distribution instances fell outside the confidence interval. Thus, even small deflections outside the confidence interval represent statistically robust effects. The resulting $\alpha$ levels will be reported.

\section{Analysis of tACS side effects}

After each experimental block featuring a given stimulation condition, participants completed a questionnaire designed to reflect (1) the perceived maximum intensity of skin sensations (itching, warmth, stinging, pulsating), phosphenes, fatigue, and pain (ranked as either "absent"/0, "light"/1, "moderate"/2, "pronounced"/3, or "strong"/4) as well as (2) the timecourse of sensations ("beginning," "end," "always"). Condition differences in perceived intensity were evaluated using Wilcoxon matched-pairs signed-rank tests without applying a correction for multiple comparisons to maximize power for detecting possibly biasing differences between conditions. Skin sensations were aggregated by computing median responses over the four qualities. To analyze whether participants were blinded or whether they could perceive the difference between sham and verum, we computed a binary score reflecting whether participants perceived peripheral sensations only in the beginning (0) or all the time (1). We report averages that can be interpreted as fractions and uncorrected $p$ values from McNemar's tests. Finally, we assessed whether significant tACS-related be- 
havioral effects detected in the main analysis could be explained by the perceived intensity of sensations. To that end, we ranked individual behavioral effects and correlated these scores with the questionnaire data by means of Spearman correlations.

\section{Analysis of EEG data}

An exhaustive description of experimental procedure and data can be found in Misselhorn et al. (2019).

\section{Participants}

Twenty-one participants (11 females; age, $23.8 \pm 2.5$ years) were invited for two sessions of EEG. None of them had a history of neurologic or psychiatric disorders. All participants gave informed written consent and received monetary compensation for their participation. The local ethics committee approved the study, which was conducted in accordance with the Declaration of Helsinki.

\section{Experimental design}

Participants received trimodal sensory stimulation (for details, see Stimulus material) on each trial of the experiment. These trimodal stimuli contained a visual, an auditory, and a tactile component. On each trial, all components underwent a brief intensity change. That is, visual contrast, auditory loudness, and vibration strength were either increased or decreased. The task was to attend bimodal pairs [visual-tactile (VT) or audio-visual (AV)] blockwise and compare attended intensity changes. These changes could be either congruent (i.e., in the same direction) or incongruent (i.e., in different directions); the respective third modality had to be ignored. Participants responded verbally after stimulus offset. Blocks of VT and AV attention contained 64 trials with equal contributions of the eight possible stimulus configurations of increases and decreases across modalities. On 2 separate days, 10 blocks each of VT and AV attention were performed in an alternating fashion, summing up to 1280 trials.

\section{Stimulus material}

Visual stimulation consisted of a circular, expanding grating presented centrally on a CRT screen (refresh rate, $120 \mathrm{~Hz}$; model HM204DTA, liyama) with gray background spanning a visual angle of $5^{\circ}$. The auditory stimulus component was a complex sinusoidal tone (13 sine waves: 64 $\mathrm{Hz}$ and its first 6 harmonics as well as $91 \mathrm{~Hz}$ and its first five harmonics; low-frequency modulator, $0.8 \mathrm{~Hz}$ ) played back with audiometric insert earphones binaurally at 70 dB (E-A-RTONE 3A, 3M). The tactile component was a high-frequency vibration delivered to the fingertips of both index fingers $(250 \mathrm{~Hz}$ on $\mathrm{C} 2$ tactors, Engineering Acoustics). Visual contrast, auditory loudness, and vibration amplitude were experimentally modulated. In total, trimodal stimuli had a fixed duration of $2 \mathrm{~s}$, and changes in intensity lasted for $300 \mathrm{~ms}$. Transitions were smoothed with cosine tapers, and onsets were jittered across trials between 700 and $1000 \mathrm{~ms}$ after stimulus onset. The magnitude of change per modality and change direction was estimated individually with a psychometric step function before experimental blocks on each day (Watson and Pelli, 1983).

\section{Processing of EEG data}

High-density EEG was recorded from 128 channels using active $\mathrm{Ag} / \mathrm{AgCl}$ electrodes referenced to the nose (EasyCap) via BRAINAMP MR amplifiers (Brain Products) and digitized after analog filtering by the amplifier (hardware settings: low cutoff, $10 \mathrm{~s}$ (time constant); high cutoff, $450 \mathrm{~Hz}$; sampling rate, $1000 \mathrm{~Hz}$ ). After resampling to 500 $\mathrm{Hz}$, data were filtered using the default settings of the EEGLAB function pop_eegfiltnew.m, which uses Hamming window sinc FIR (finite impulse response) filters and estimates the filter order [bandpass, $30-120 \mathrm{~Hz}$ (order 220); notch, $49-51 \mathrm{~Hz}, 99-101 \mathrm{~Hz}$ (order 1650); Delorme and Makeig, 2004]. Due to low signal-to-noise ratio, 19 electrodes of the outer rim covering neck and chin were excluded from further analysis. Before preprocessing, data were rereferenced to the common average and cut into epochs locked to stimulus onset ( -500 to $2000 \mathrm{~ms}$ ). Due to $1 / \mathrm{f}$ properties of cortical activity, an EEG signal $>30 \mathrm{~Hz}$ is usually dominated by muscle activity. Thus, we used independent component (IC) analysis to identify components of cortical gamma activity by evaluating topography, time course, and spectrum of all ICs (Hipp and Siegel, 2013). Additionally, we identified ICs related to muscle activity underlying miniature saccades (Hassler et al., 2011). We rejected all components that did not show clear characteristics of cortical gamma activity as well as the ICs reflecting miniature saccades (rejected ICs, $66 \pm 11 \%)$. Stratified data held, on average, $426 \pm 89$ epochs per participant. We divided data for each participant into two sets based on the congruence of changes in the visual and tactile stimuli. That is, irrespective of attention condition, congruent trials featured trimodal stimuli in which the visual and tactile components changed in the same direction. Conversely, incongruent trials contained all trials with stimuli in which the visual and tactile components changed in different directions. In sensor space, event-related potentials were averaged per experimental condition and subtracted from single-trial data. Source reconstruction was performed with exact low-resolution electromagnetic tomography (eLORETA; regularization, 0.05; Pascual-Marqui et al., 2011). Spatial filters were constructed using a three-shell head model (Nolte and Dassios, 2005) and a cortical grid in MNI space obtained by downsampling the Freesurfer template to 10,000 grid points (Desikan et al., 2006). Dipole directions were chosen by finding the direction of maximum power at $40 \mathrm{~Hz}$, with singular value decomposition for each node in the cortical surface. Power was estimated by computing auto-spectra using fast Fourier transform (fft.m function in Matlab) based on all trials of a given condition and participant.

\section{Statistical analysis}

To compute the time courses of power and the coherence of sensory gamma oscillations, we used a sliding window approach with Hanning windows of length $1 \mathrm{~s}$, which were shifted in $50 \mathrm{~ms}$ steps from -1000 to $1000 \mathrm{~ms}$ relative to change onset. In the resulting 21 windows, we computed cross-spectra for the whole cortical grid from each trial and subsequently averaged them. From the trial-averaged cross-spectra, we computed imaginary co- 
herence (iCoh; Nolte et al., 2004) among all cortical nodes as well as power at each node at $40 \mathrm{~Hz}(1 \mathrm{~Hz}$ resolution). Finally, power was averaged across all nodes of the left and right visual and somatosensory cortices based on the anatomic atlas of Freesurfer (Desikan et al., 2006). iCoh was averaged for all intrahemispheric connections between visual and somatosensory cortices ignoring all intraregional as well as interhemispheric connections. Each individual time course of power or iCoh was normalized by z-transform based on all data points of a given time course. Additionally, we subtracted a baseline from -500 to -250 ms relative to change onset (note: time points are referred to by their center bin). Statistical comparison with baseline and between conditions was performed by averaging time course data for the time points of change $(0-300 \mathrm{~ms})$ and computing one-sample $t$ tests within conditions and paired-sample $t$ tests across conditions. No correction for multiple tests was applied to have maximal power for detecting possibly biasing differences in power. iCoh was analyzed similarly by averaging for the epoch of change $(0-300 \mathrm{~ms})$ and performing $t$ tests as described before. Here, we corrected for multiple testing according to Bonferroni.

\section{Results}

\section{ANOVA on behavioral outcomes during tACS}

A complete repeated measures ANOVA model with factors HEMISPHERE (same/different), FREQUENCY (alpha/gamma), STIMULATION (sham/in-phase/anti-phase), and CONGRUENCE (congruent/incongruent) was computed for both accuracy and RTs. Participants were well trained on the task and gave, on average, correct responses in $\sim 83 \%$ of all trials. Accuracy differed significantly between congruent and incongruent trials $\left(F_{(1,19)}=\right.$ $10.122, p=0.005, \eta_{p}^{2}=0.348$ ), and errors were less likely in congruent trials $(85.48 \%)$ when compared with incongruent trials $(80.36 \%)$. No other factor or any interaction significantly influenced accuracy (Extended Data Table 1-1). The timing of responses broken down by condition is shown in Figure 2A. RTs showed a similar, but stronger effect of CONGRUENCE (Fig. 2E; $F_{(1,19)}=34.659, p=$ $\left.1.142 * 10^{-5}, \eta_{p}^{2}=0.646\right)$. That is, responses in congruent trials were on average faster than in incongruent trials (mean RT difference, $105 \mathrm{~ms}$ ). In contrast to accuracy, all other factors significantly affected RTs, resulting in significant two-, three-, and four-way interactions (Table 1, Extended Data Table 1-1). To resolve these high-order interactions, we computed reduced ANOVA models until interpretable two-way interactions remained.

First, we resolved the factor HEMISPHERE by computing separate ANOVAs with factors FREQUENCY, STIMU$\angle A T I O N$, and CONGRUENCE. For stimulation over the hemisphere not targeted by sensory stimulation (DIFFERENT), we found a significant effect of CONGRUENCE $\left(F_{(1,19)}=24.803, p=8.308 * 10^{-5}, \eta_{p}^{2}=0.566\right)$ as well as an interaction between FREQUENCY and STIMULATION $\left(F_{(2,38)}=3.771, p=0.038, \eta_{p}^{2}=0.166\right)$. Post hoc comparisons revealed opposing effects for alpha and gamma stimulation, as follows: under alpha stimulation, RTs shortened from sham to both in-phase and anti-phase stimulation. Under gamma stimulation, RTs were prolonged from sham to both in-phase and anti-phase stimulation. None of these differences, however, were significant after correction for multiple comparisons (all $p$ $>0.2$; Fig. 2G).

For stimulation over the hemisphere that was targeted by sensory stimulation (SAME), we found a significant effect of CONGRUENCE $\left(F_{(1,19)}=40.028, p=4.518 *\right.$ $\left.10^{-6}, \eta_{p}^{2}=0.678\right)$ as well as an interaction among FREQUENCY, STIMULATION, and CONGRUENCE $\left(F_{(2,38)}=\right.$ $\left.7.548, p=0.002, \eta_{p}^{2}=0.284\right)$. This three-way interaction was investigated by the resolving factor FREQUENCY. For alpha frequency, only CONGRUENCE significantly affected RTs $\left(F_{(1,19)}=43.454, p=2.614 * 10^{-6}, \eta_{p}^{2}=\right.$ 0.696). In contrast, a reduced ANOVA for gamma frequency showed, next to an effect of CONGRUENCE $\left(F_{(1,19)}=26.133, p=6.194 * 10^{-5}, \eta_{p}^{2}=0.579\right)$, an interaction between STIMULATION and CONGRUENCE $\left(F_{(2,38)}=4.578, p=0.018, \eta_{p}^{2}=0.194\right)$. Post hoc comparisons showed significant effects of CONGRUENCE under all stimulation conditions (Bonferroni-corrected $p$ values; sham: $t_{(19)}=-5.099, p=0.0002$; in-phase: $t_{(19)}=$ -3.516, $p=0.0069$; anti-phase: $t_{(19)}=-3.049, p=$ 0.0198). Pairwise comparisons between stimulation conditions showed that the effect of CONGRUENCE was significantly smaller under in-phase compared with sham stimulation (Bonferroni-corrected $p$ values; sham vs inphase: $t_{(19)}=-2.630, p=0.0495$; sham vs anti-phase: $t_{(19)}=-2.325, p=0.0939$; in-phase vs anti-phase: $t_{(19)}=$ $0.5338, p=1.7991)$. Specifically, responses to congruent stimuli were delayed while responses to incongruent stimuli did not show significant differences to sham (Fig. 2F).

\section{Nonparametric follow-up analysis of response times distributions}

Significant two-way interactions were followed up by comparisons of CDFs of RT data that were evaluated using nonparametric permutation statistics. This approach represents a powerful investigation of subtle changes in the shape of distributions that do not necessarily result in significant changes of mean values. It should be noted that correction for multiple comparisons was performed for both the number of condition-wise comparisons and the range of RTs, resulting in a conservative alpha value (alpha $=0.000129$ ). Condition differences exceeding the confidence interval, even for narrow $\mathrm{RT}$ ranges, thus represent statistically robust effects.

The interaction between STIMULATION and CONGRUENCE for stimulation of the hemisphere targeted by sensory input (SAME) was followed up by subtracting CDFs of stimulation conditions pairwise separately for congruent and incongruent trials. For congruent trials, both inphase and anti-phase stimulation differed significantly from sham, meaning that responses were slowed down by stimulation (sham vs in-phase: $440-960$ and $1330-$ 1660 ms; sham vs anti: $880-1610$ ms; Fig. $3 A$, middle). Over and above the effects detectable by ANOVA, we found a difference between in-phase and anti-phase stimulation (430-710 ms; Fig. 3A, middle). Accordingly, inphase stimulation slowed responses more strongly when 
A

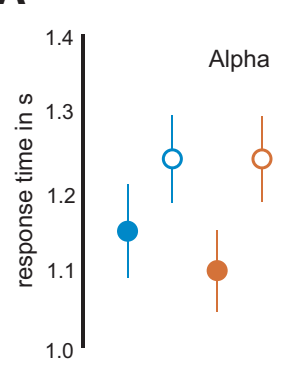

B

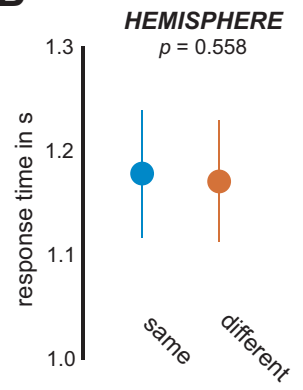

$\mathbf{F}$

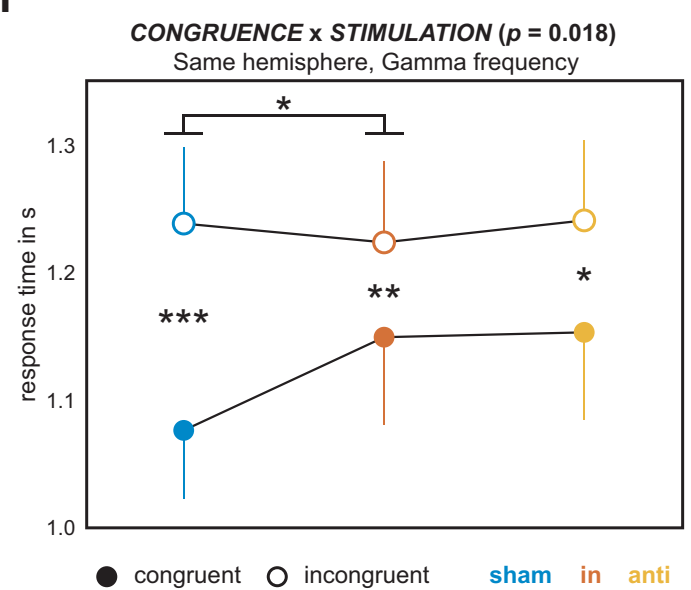

Same hemisphere

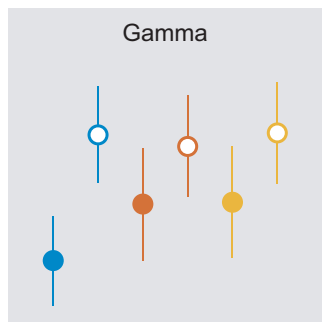

congruent $\mathrm{O}$ incongruent

C

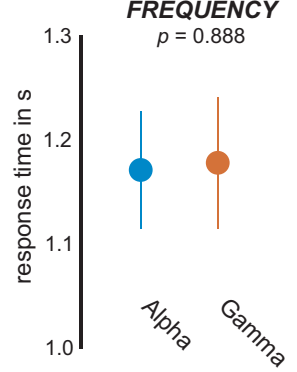

D

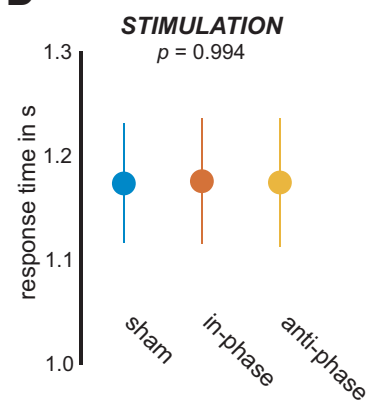

Different hemispheres

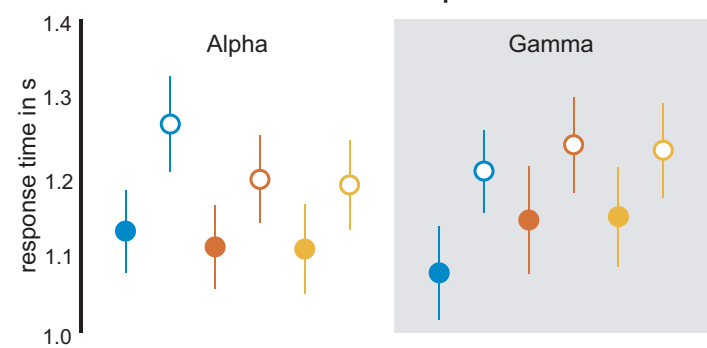

sham in-phase anti-phase

E

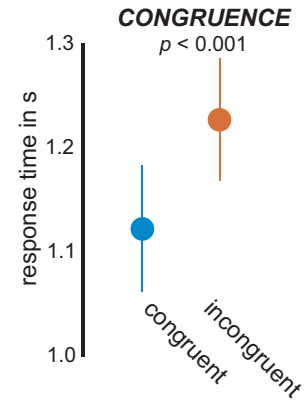

G

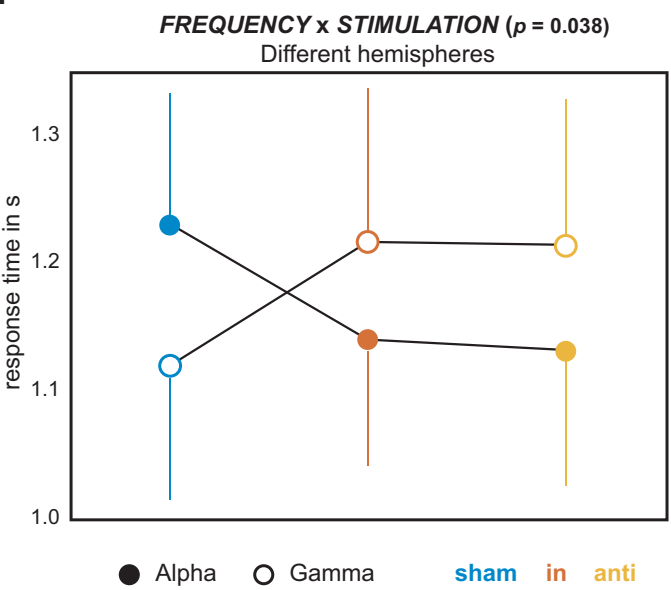

Figure 2. Results from ANOVA. A correlation analysis between tACS side effect and significant interactions in the RT ANOVA is provided as Extended Data Figure 2-1. A, Overview of all conditions entering the HEMISPHERE (2) $\times$ FREQUENCY (2) $\times$ STIMULATION (3) × CONGRUENCE (2); repeated-measures ANOVA (rmANOVA). Filled/empty circles represent congruent/incongruent trials. Factor levels of STIMULATION are color coded (blue, sham; red, in-phase; yellow, anti-phase). B-E, Main effects resulting from $2 \times 2 \times 3 \times 2$ rm-ANOVA. $\boldsymbol{F}$, CONGRUENCE $\times$ STIMULATION interaction for gamma stimulation over the hemisphere targeted by sensory stimuli $(* p<0.05, * * p<0.01$, ***p $<0.001)$. G, FREQUENCY $\times$ STIMULATION interaction for stimulation of the hemisphere not targeted by sensory stimulation.

compared with anti-phase stimulation. The same analysis for incongruent trials did not show any differences with respect to stimulation conditions (Fig. $3 A$, right).

The interaction between FREQUENCY and STIMULA$T I O N$ for stimulation of the hemisphere not targeted by sensory input (DIFFERENT) was followed up by subtracting CDFs of stimulation conditions pairwise separately for alpha and gamma stimulation. For alpha stimulation, we found significant differences between sham and in-phase stimulation as well as between sham and anti-phase stimulation corresponding to a speeding of responses (sham vs in-phase: $180-670$ and $1330-2280 \mathrm{~ms}$; sham vs anti- phase: $450-690$ and $1010-2215 \mathrm{~ms}$; Fig. 3B, middle). For gamma stimulation, we found significant differences between sham and in-phase as well as sham and anti-phase stimulation corresponding to a slowing of responses (sham vs in-phase: $525-970$ and $1330-2240 \mathrm{~ms}$; sham vs anti-phase: 865-1015 and 1615-2040 ms; Fig. 3B, right). For both frequencies, we did not find significant differences between in-phase and anti-phase stimulation.

\section{Side effects of tACS}

Most participants reported tACS-related side effects (Extended Data Fig. 2-1). While most participants re- 
Table 1: Significant effects of all computed ANOVA models on response times

\begin{tabular}{|c|c|c|c|}
\hline Factor & $F$ & $p$ & $\eta_{p}^{2}$ \\
\hline \multicolumn{4}{|l|}{ Complete ANOVA model } \\
\hline CONGRUENCE & 34.659 & 0.000 & 0.646 \\
\hline STIMULATION $\times$ CONGRUENCE & 4.199 & 0.032 & 0.181 \\
\hline FREQUENCY $\times$ STIMULATION $\times$ CONGRUENCE & 4.089 & 0.027 & 0.177 \\
\hline HEMISPHERE $\times$ FREQUENCY $\times$ STIMULATION $\times$ CONGRUENCE & 4.862 & 0.015 & 0.204 \\
\hline \multicolumn{4}{|l|}{ Reduced model: different HEMISPHEREs } \\
\hline CONGRUENCE & 24.803 & 0.000 & 0.566 \\
\hline FREQUENCY $\times$ STIMULATION & 3.771 & 0.038 & 0.166 \\
\hline \multicolumn{4}{|l|}{ Reduced model: same HEMISPHERE } \\
\hline CONGRUENCE & 40.028 & 0.000 & 0.678 \\
\hline FREQUENCY $\times$ STIMULATION $\times$ CONGRUENCE & 7.548 & 0.002 & 0.284 \\
\hline \multicolumn{4}{|l|}{ Reduced model: same HEMISPHERE, alpha FREQUENCY } \\
\hline CONGRUENCE & 43.454 & 0.000 & 0.696 \\
\hline \multicolumn{4}{|l|}{ Reduced model: same HEMISPHERE, gamma FREQUENCY } \\
\hline CONGRUENCE & 26.133 & 0.000 & 0.579 \\
\hline STIMULATION $\times$ CONGRUENCE & 4.578 & 0.018 & 0.194 \\
\hline
\end{tabular}

Complete tables of ANOVA for accuracy and response time data can be found as Extended Data Table 1-1.

ported "light" to "strong" skin sensations (median \pm interquartile range, $1 \pm 1.25)$ only three participants reported phosphenes $(0 \pm 0)$. Fatigue $(0 \pm 1)$ and pain $(0$ $\pm 1)$ were absent in the majority of participants. Importantly, the intensity of sensations overall did not differ with respect to sham, in-phase, or anti-phase stimulation (uncorrected, for all, $p>0.09$ ) and also showed no differences with respect to stimulation frequency (uncorrected, for all, $p>0.38$ ). Next to the intensity of sensations, we asked for the timecourse of a given sensation and coded responses into a binary decision for initial (0) or constant (1) stimulation (averages; $10 \mathrm{~Hz}$ : sham $=0.32$, in-phase $=$ 0.68 , anti-phase $=0.74 ; 40 \mathrm{~Hz}$ : sham $=0.32$, in-phase $=$ 0.68 , anti-phase $=0.63$ ). Differences in the timecourse of perception indicative of sham and verum conditions were found significant or trending for both alpha and gamma stimulation (all uncorrected; alpha: sham vs in-phase, $p=$ 0.03 ; sham vs anti-phase, $p=0.02$; in-phase vs antiphase, $p=0.87$; gamma: sham vs in-phase, $p=0.03$; sham vs anti-phase, $p=0.07$; in vs anti-phase, $p=0.87$ ). Sorensen-Dice similarity coefficients showed that ratings for alpha and gamma stimulation were comparable (sham, 1; in-phase, 0.77; anti-phase, 0.77). Finally, correlations among the average effect values of all three significant interactions detected with ANOVA and the overall intensity of skin sensations were weak and nonsignificant (all $|r|$ $<0.25$ and all $p>0.3$ ). Interestingly, extreme values for skin sensations were more likely to occur in participants with weak behavioral effects. Nonsignificant correlations thus showed opposite signs than would have been expected if the strength of sensations was indicative of behavioral effect size.

\section{EEG data}

A connectivity analysis of EEG data previously recorded during a similar task was used to guide interpretation of the tACS effects on behavior (Misselhorn et al., 2019). Time series of cleaned EEG data were projected to source space to analyze local and interareal synchronization of gamma oscillations at $40 \mathrm{~Hz}$ in and between early visual and somatosensory regions. First, we analyzed power to identify potential biases to the evaluation of iCoh (Nolte et al., 2004). All statistics of control analyses are reported without correction for multiple comparisons to have maximal power for the detection of potentially small, but biasing differences in power. During the change interval, condition-averaged $40 \mathrm{~Hz}$ gamma power was relatively increased in the right hemisphere and, to a smaller degree, suppressed or unchanged in the left hemisphere (Fig. 4A, middle). Statistical analysis of power change in the visual and somatosensory regions of interest (ROIs) showed that these trends were not significant (Extended Data Fig. 4-1; left visual: $t_{(20)}=-1.506, p=0.148$; left somatosensory: $t_{(20)}=-0.891, p=0.384$; right visual: $t_{(20)}=0.561, p=0.581$; right somatosensory: $t_{(20)}=$ 1.147, $p=0.265)$. Furthermore, power changes did not differ significantly between hemispheres (visual left-right: $t_{(20)}=-1.292, p=0.211$; somatosensory left-right: $t_{(20)}=$ $-1.724, p=0.100)$. Also, condition-averaged $40 \mathrm{~Hz}$ power did not correlate with condition-averaged iCoh for left and right visual ROls as well as left somatosensory ROI (Extended Data Fig. 4-2; left visual: $r_{(19)}=-0.368, p$ $=0.111$; right visual: $r_{(19)}=-0.145, p=0.532$; left somatosensory: $\left.r_{(19)}=-0.199, p=0.388\right)$, but showed a negative correlation for right somatosensory ROI (Extended Data Fig. 4-2; right somatosensory: $r_{(19)}=-0.532$, $p=0.013$ ). Importantly, statistical comparison between congruent and incongruent trials did also not show any significant differences (Fig. 4A: I, left somatosensory: congruent, $t_{(20)}=-0.241, p=0.812$; incongruent, $t_{(20)}=$ $-0.848, p=0.407$; congruent-incongruent, $t_{(20)}=0.396$, $p=0.697$; II, left visual: congruent, $t_{(20)}=-1.084, p=$ 0.291 ; incongruent, $t_{(20)}=-0.613, p=0.547$; congruentincongruent, $t_{(20)}=-0.259, p=0.798$; III, right somatosensory: congruent, $t_{(20)}=0.001, p=0.999$; incongruent, $t_{(20)}=1.592, p=0.127$; congruent-incongruent, $t_{(20)}=$ $-1.070, p=0.298$; IV, right visual: congruent, $t_{(20)}=$ 1.151, $p=0.263$; incongruent, $t_{(20)}=-0.541, p=0.595$; congruent-incongruent, $\left.t_{(20)}=1.348, p=0.193\right)$. As a next step, we analyzed iCoh in a left and right intrahemispheric network between visual and somatosensory cortices, respectively (Fig. 4A, arrows). In the left hemisphere, 

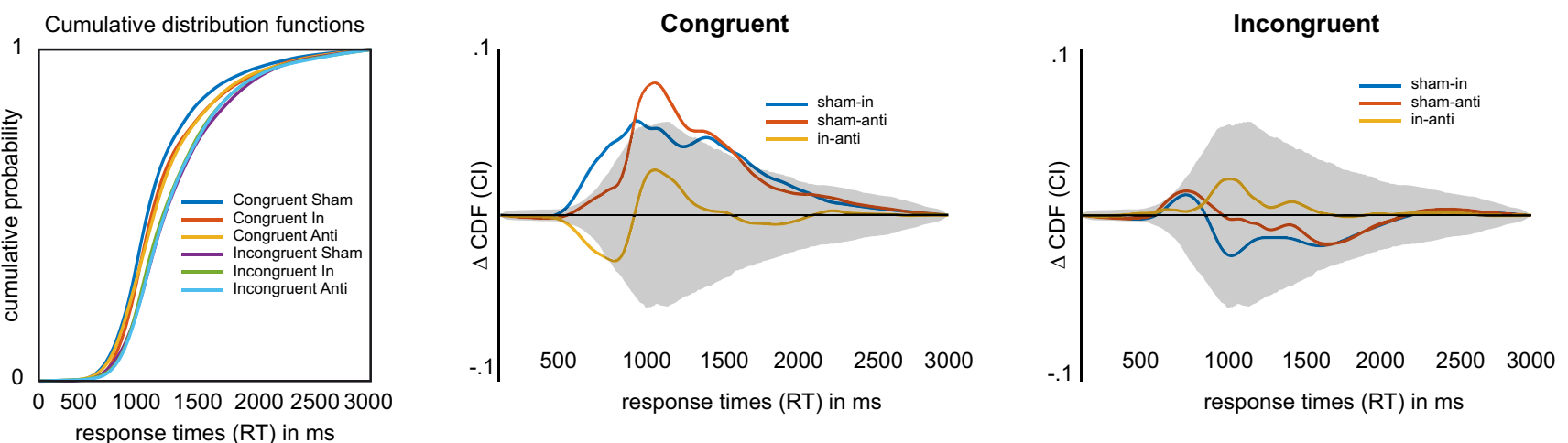

B

Post-hoc interaction FREQUENCY x STIMULATION (Different hemispheres)
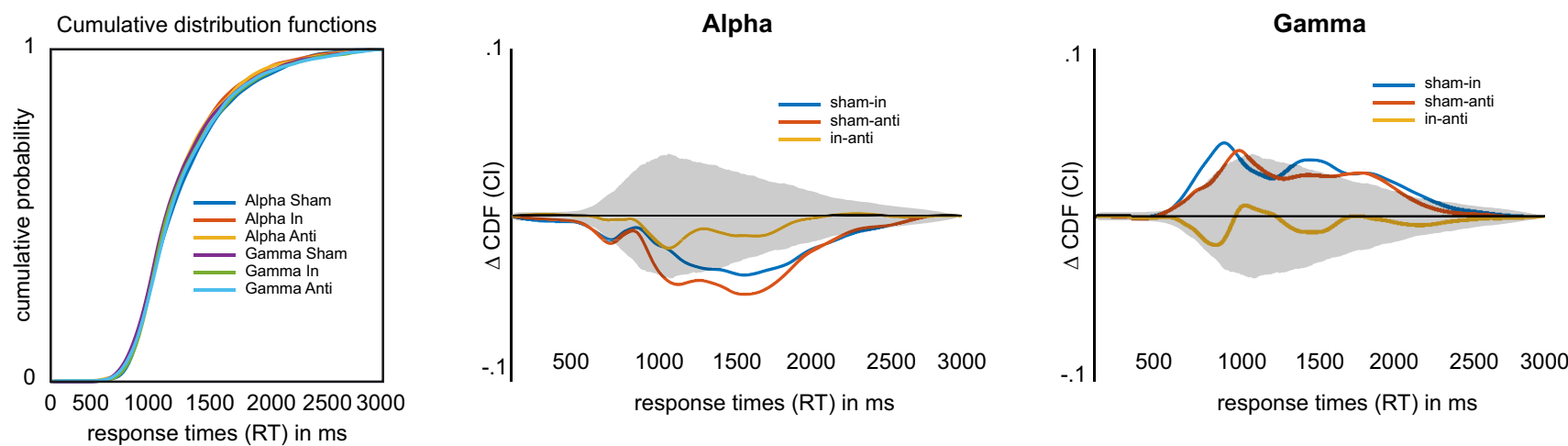

Figure 3. Results from follow-up analysis comparing RT distributions. $\boldsymbol{A}$, Follow-up analysis of the STIMULATION $\times$ CONGRUENCE interaction found for gamma stimulation over the hemisphere targeted by sensory input (same). Left, CDFs corresponding to all levels of the $2 \times 3$ interaction. Middle, Differences in CDFs for congruent trials between levels of factor STIMULATION. Gray-shaded area indicates the $\mathrm{Cl}$ as estimated by nonparametric permutation statistics (corrected for multiple comparisons, $p<0.000129$ ). All differences outside the $\mathrm{Cl}$ indicate significant differences between the respective conditions. Right, Differences in CDFs for incongruent trials between levels of factor STIMULATION. B, Follow-up analysis of the FREQUENCY $\times$ STIMULATION interaction found for stimulation over the hemisphere not targeted by sensory input. Left, CDFs corresponding to all levels of the $2 \times 3$ interaction. Middle, Differences in CDFs for trials under alpha stimulation between levels of factor STIMULATION. Gray-shaded area indicates the $\mathrm{Cl}$ as estimated by permutation statistics (corrected for multiple comparisons, $p<0.000129$ ). Right, Differences in CDFs for trials under gamma stimulation between levels of factor STIMULATION.

we observed a decrease in iCoh for congruent, but not for incongruent trials, resulting in a significant difference between congruent and incongruent conditions (Fig. 4B, left; Bonferroni-corrected $p$ values; congruent, $t_{(20)}=-4.287$, $p=0.001$; incongruent, $t_{(20)}=-0.130, p=2.694$; congruent-incongruent, $\left.t_{(20)}=-2.882, p=0.027\right)$. In the right hemisphere, iCoh did not significantly change for either congruent or incongruent trials (Fig. $4 B$, right; Bonferroni-corrected $p$ values; congruent, $t_{(20)}=0.621, p$ $=1.624$; incongruent, $t_{(20)}=0.363, p=2.162$; congruentincongruent, $\left.t_{(20)}=0.177, p=2.585\right)$.

\section{Discussion}

We investigated the role of long-range gamma synchronization between sensory cortices in multisensory perception. In a cross-modal matching task, participants compared amplitude information across visual and somatosensory modalities, which we assumed to rely on direct interaction between sensory cortices. In an attempt to modulate such direct cross talk between the senses, we used multi-electrode tACS to influence coupling between sensory gamma oscillations in a phase-specific as well as a frequency-specific manner. We show that gamma tACS, but not alpha tACS, over sensory cortices targeted by sensory input modulated the degree of multisensory congruence enhancement. Stimulation over the same areas in the hemisphere not targeted by sensory stimulation did not have a significant influence on congruence enhancement, but showed opposite effects for alpha and gamma stimulation. Finally, we reanalyzed EEG data from a comparable task and found imaginary coherence between visual and somatosensory cortices to be modulated by cross-modal congruence.

\section{Cross-modal matching involves communication, not feature binding, between modalities}

In our paradigm, cross-modal matching between congruent stimuli was associated with speeded responses when compared with the matching of incongruent stimuli. This behavioral benefit of cross-modal congruence is well 
A
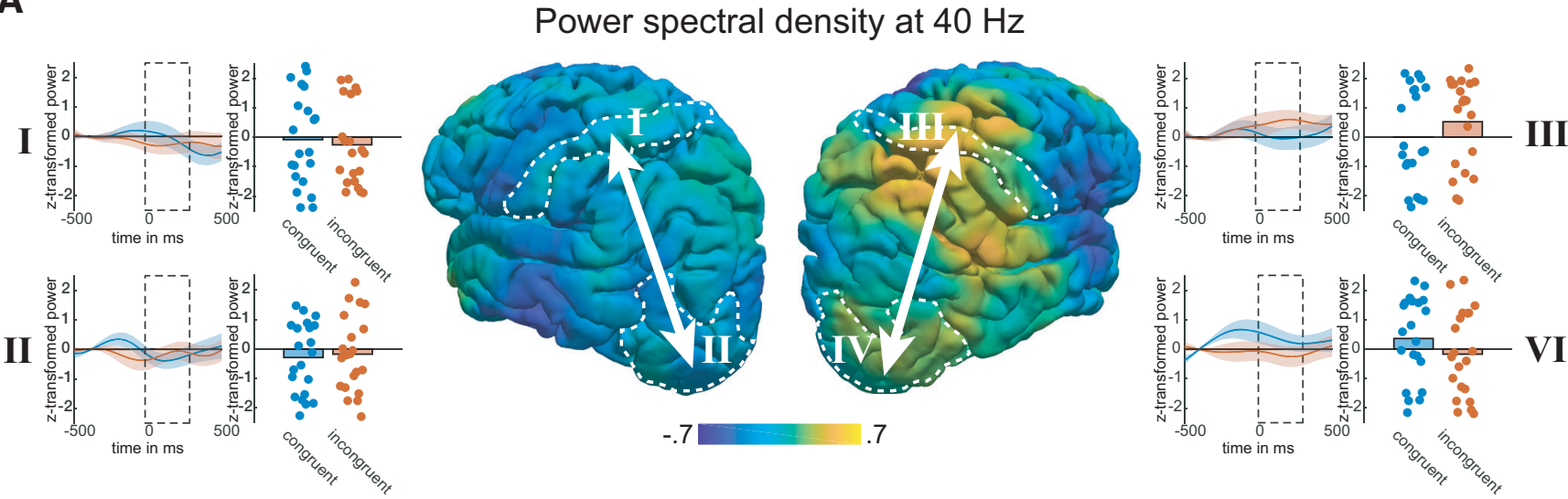

B

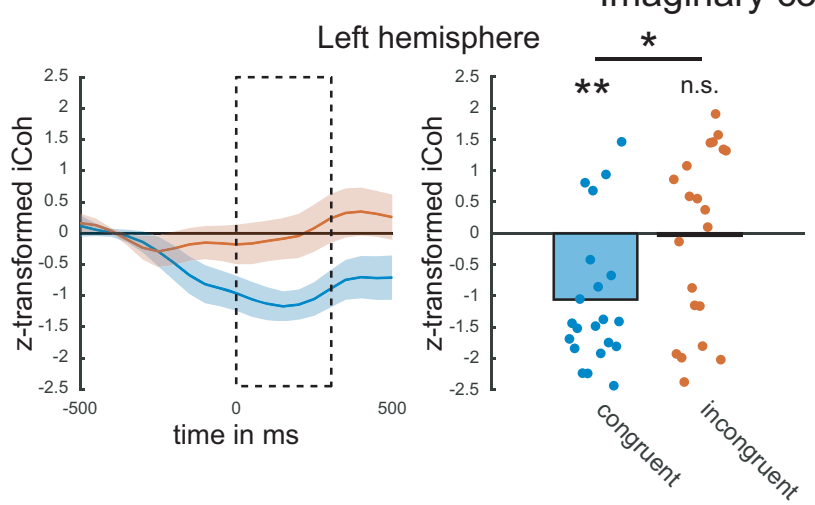

Imaginary coherence at $40 \mathrm{~Hz}$

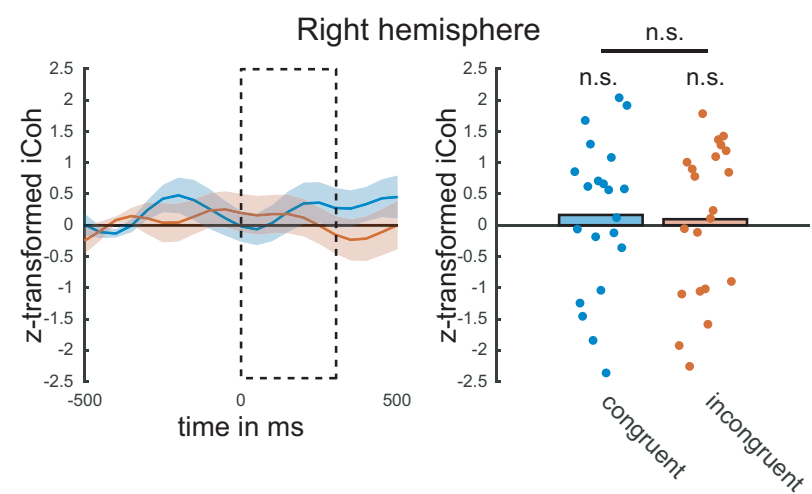

Figure 4. Results from reanalysis of EEG data. In a previous study (Misselhorn et al., 2019), EEG was recorded during a similar experimental paradigm. Here, we reanalyzed the data with a focus on power and imaginary coherence in and between visual and somatosensory areas at $40 \mathrm{~Hz}$. In the extended data, we present an analysis comparing the average change in $40 \mathrm{~Hz}$ power across hemispheres (Extended Data Fig. 4-1) as well as an analysis of the correlation between power and iCoh (Extended Data Fig. 4-2). $A$, Middle, Distribution of average normalized power at $40 \mathrm{~Hz}$ during the change interval. Dotted outlines indicate borders of ROls. Left, right, Roman numerals indicate the ROI in which power was computed. Each graph depicts the time course of power changes for congruent (blue) and incongruent (red) trials as well as the average power during change (dotted box) as a bar/scatter plot. All comparisons resulted in nonsignificant differences (all $p>0.05$ ). $\boldsymbol{B}$, Left, Timecourse of iCoh between visual (II) and somatosensory (I) cortices of the left hemisphere. Bar/scatter plots depict average iCoh during change interval (dotted box; n.s. $p>0.05, * p<0.05$, $* * p<0.01$ ). Right, Timecourse of iCoh between visual (IV) and somatosensory (III) cortices of the right hemisphere. Bar/scatter plots depict average iCoh during change interval.

in line with previous studies that consistently showed faster responses and elevated accuracy of detecting or discriminating congruent multisensory stimuli (Bolognini et al., 2005; Schneider et al., 2008b; Göschl et al., 2014; Misselhorn et al., 2016). It was proposed that these behavioral benefits might arise because of enhanced crossmodal binding mediated by elevated synchrony between sensory gamma oscillations (Senkowski et al., 2008; Keil and Senkowski, 2018). Accordingly, congruent multisensory stimuli should induce stronger cross-modal coupling when compared with incongruent stimuli, and synchronizing sensory cortices by tACS should be beneficial for the processing of congruent, but not incongruent, multisensory inputs. In our data, however, we found the opposite pattern of results. Compared with sham stimulation, inphase gamma stimulation over cortices targeted by sensory input led to a significant reduction of the congruence effect. Specifically, responses in congruent trials were slowed down, whereas responses in incongruent trials were as fast as under sham stimulation. While anti-phase gamma stimulation showed a similar pattern, this effect was not significant on the level of the ANOVA. In a follow-up analysis using nonparametric permutation statistics to compare CDFs of RT data, we found both inphase and anti-phase gamma stimulation over taskrelevant sensory cortices to delay responses to congruent, but not incongruent, stimuli. Interestingly, inphase stimulation showed an earlier peak of difference with sham stimulation than with anti-phase stimulation, leading to a significantly stronger delay of responses for in-phase compared with anti-phase tACS for responses between $\sim 400$ and $\sim 700 \mathrm{~ms}$.

Additionally, we analyzed cortical $40 \mathrm{~Hz}$ activity in EEG data that we had previously recorded during a similar task (Misselhorn et al., 2019). In a source space analysis, we found condition-averaged power to be relatively suppressed in the left hemisphere and relatively increased in the right hemisphere. Although these global trends were 
also reflected in visual and somatosensory cortices, statistical comparison did not show significant changes in power in either left or right hemisphere. Furthermore, power was not correlated with coupling in the left visual and somatosensory cortices as well as in right visual cortex. Only power in right somatosensory cortex showed a negative correlation with imaginary coherence. Importantly, we did not find significant modulations of power by cross-modal congruence. In the analysis of coupling in the right hemisphere, we could not detect any effect of cross-modal congruence. This absence of modulation in $40 \mathrm{~Hz}$ iCoh, however, might be confounded by the negative correlation between iCoh and power and is not interpreted (Bastos and Schoffelen, 2016). Importantly, we could show that $40 \mathrm{~Hz}$ iCoh between visual and somatosensory cortices of the left hemisphere was significantly decreased during cross-modal matching for congruent stimuli, but not for incongruent stimuli.

This pattern of results cannot be explained with the binding-by-synchrony hypothesis (Engel et al., 2001; Senkowski et al., 2008). In this view, synchrony serves as a tag that binds neuronal assemblies that code for the same, potentially multisensory, object or feature. Behavioral benefits would thus arise because of a strengthened or more stable representation of the multisensory object that facilitates further processing. This rather passive or static perspective on cross-modal interactions does not seem to apply to the paradigm used here, where active matching between the senses was required. We thus propose to interpret our results in the context of the communication through coherence hypothesis, which represents an extension of the binding-by-synchrony hypothesis by highlighting the dynamic routing capabilities of synchronizing distant cell assemblies (Fries, 2009, 2015). In this perspective, coherence between gamma oscillations enables communication between interconnected cortical areas that allows for information transfer. We speculate that perceiving incongruent stimuli was associated with an increased need for communication between sensory areas reflected by stable levels of crossmodal gamma coherence over time, which were seen in the reanalysis of EEG data. Congruent stimuli, on the other hand, were related to a fast decoupling between sensory cortices, which would correspond to a termination of direct communication, perhaps to the effect of gating information flow to higher-order cortical areas such as the parietal lobes. Synchronizing sensory cortices by $40 \mathrm{~Hz}$ tACS is proposed to have hampered such sensory decoupling and thereby reduced the amount of behavioral benefit for congruent stimuli. Collectively, these findings lend support to the idea that gamma oscillations play a critical role in structuring interactions and likely communication among sensory cortical areas.

\section{Limited phase specificity of behavioral tACS effects}

As described above, we found in-phase gamma stimulation to exhibit significant effects on processing crossmodally congruent stimuli in an ANOVA. In a follow-up analysis, however, we show that in-phase and anti-phase stimulation differ in a small fraction of the RT distribution quantitatively, but not as hypothesized qualitatively. That is, anti-phase gamma stimulation also reduced the behavioral benefit of cross-modal congruence. This result stands in contrast to earlier findings that describe differential effects of in-phase and anti-phase stimulation on cortical coupling and/or behavior (Polanía et al., 2012; Helfrich et al., 2014; Schwab et al., 2019). One explanation for these phase-unspecific effects might be that tACS boosted the power of ongoing sensory gamma oscillations without deflection of phase. While, in principle, this is possible, the current pattern of results is unlikely to be related to enhanced gamma power. First, the power of sensory gamma oscillations was previously shown to be associated with faster response times for both visual and tactile stimuli (Krebber et al., 2015; van Es and Schoffelen, 2019). Thus, enhanced sensory gamma power should speed, not delay, responses. Second, cross-modally congruent stimuli have been repeatedly shown to induce stronger gamma responses than incongruent stimuli (Senkowski et al., 2005, 2007; Yuval-Greenberg and Deouell, 2007; Schneider et al., 2008a; Krebber et al., 2015). Thus, further boosting of gamma power should have aided not hindered responding to congruent stimuli.

Alternatively, we should question the overarching assumptions that were made in this and many other tACS studies designed to modulate interareal coupling (Saturnino et al., 2017). That is, expecting effects of opposite sign for in-phase and anti-phase stimulation relies on a number of assumptions that might not necessarily hold, most importantly that (1) the targeted process actually exhibits (near) zero-phase properties and that (2) cortical activity can be affected by tACS without phase lag. The targeted process here is direct communication between sensory areas, which has been described, but remains poorly understood (Cappe and Barone, 2005; Kayser et al., 2008). Importantly, long-range interactions between distinct sensory cortices involve conduction delays that might differ with respect to task parameters, rendering (near) zero-lag coupling unlikely (Raij et al., 2010). Furthermore, tACS might not affect cortical activity with the same phase lag over participants (Riecke et al., 2015; Asamoah et al., 2019). Although electric currents reach cortical structures without significant temporal lag, differences in the anatomy of the skull and, most importantly, differences in cortical folding might lead to different preferred phases of tACS (Liu et al., 2018). That is, cortical target orientations might differ by up to $180^{\circ}$ across participants, which would render effective in-phase and anti-phase stimulation to be opposite for extreme cases. In our data, we suggest that the lack of phase specificity might have arisen due to suboptimal fit of individual phase lags with the $0^{\circ}$ and $180^{\circ}$ conditions of stimulation that were used. In effect, both in-phase and anti-phase stimulation might have suited some, but not all, participants' anatomy and physiology. Testing this speculation, however, requires a larger set of phase lag conditions and, optimally, physiologic data to relate to. 


\section{Contralateral alpha/gamma dynamics regulate cortical excitability}

To control for unspecific effects of tACS, we used stimulation over visual and somatosensory areas in the hemisphere not targeted by lateralized sensory stimuli as an active control. Our results showed global modulations of response times that were specific with respect to frequency, but unspecific with respect to the task conditions. Note, however, that these effects were small and were significant only when evaluating RT distributions with permutation statistics. While gamma stimulation showed overall slowing of responses, alpha stimulation showed overall speeding of responses. These findings can be related to the functionally opposing roles of alpha and gamma oscillations in cortex, as follows: while gamma oscillations are enhanced from activated cortical areas (Donner and Siegel, 2011), alpha oscillations predominate in task-irrelevant cortical regions (Jensen and Mazaheri, 2010). This view is supported by a negative or positive correlation of EEG gamma and alpha power, respectively, with the BOLD signal (Mulert et al., 2010; Scheeringa et al., 2011). Importantly, the level of ongoing alpha activity could be shown to be a readout of cortical excitability as determined by transcranial magnetic stimulation (Romei et al., 2008). Cortical excitability, as controlled by alpha/gamma dynamics, is also discussed as a mechanism underlying top-down control of perceptual processes (Jensen and Mazaheri, 2010; Bonnefond and Jensen, 2015). For instance, cued spatial attention led to a lateralization of prestimulus alpha power to the taskirrelevant hemisphere, while stimulus-related gamma activity was lateralized to the task-relevant hemisphere (Marshall et al., 2015). In our tACS experiment, stimuli were presented lateralized and pretrial cues were used to guide the spatial attention of participants. We propose that reducing cortical excitability of the task-irrelevant hemisphere through alpha stimulation improved processing in the task-relevant hemisphere. Conversely, gamma stimulation might have increased the excitability of the task-irrelevant hemisphere and thereby impeded processing in the task-relevant hemisphere. A similar result has recently been obtained for unilateral stimulation over temporoparietal cortex in a dichotic listening task (Wöstmann et al., 2018). In their study, alpha tACS decreased the recall of contralateral items while gamma tACS showed the opposite effect. Our results, thus, add to the abundant literature that suggests an important role of alpha/gamma dynamics in modulating cortical excitability.

\section{Limitations and future directions}

The main limitation, which applies to most current tACS experiments, is the lack of concurrent measurement of the underlying physiology. Due to nonlinear artifacts in the EEG, acute physiologic effects of tACS can currently not be investigated with EEG (Noury et al., 2016; Noury and Siegel, 2017). Yet, investigating aftereffects represents a viable alternative that can be used to observe the lingering effects of stimulation (Schwab et al., 2019). Due to the unavailability of these data, we cannot provide details of the physiologic underpinnings of the behavioral modulation described here. To compensate for the lack of direct physiologic data, we reanalyzed data from a similar task. Although this allows some inference and certainly can guide the interpretation of the behavioral results, its explanatory power is limited because separate groups were investigated. We therefore could not investigate any direct relations between the EEG and tACS-modulated behavior. As outlined above, another limitation was the choice of two phase lags only. Future studies should use more phase lags to identify optimal phase lags that likely differ across individuals.

\section{Conclusions}

While there is ample evidence for the active role of gamma oscillations for interactions within sensory streams, we provide evidence for the importance of coordinated sensory gamma oscillations for direct crossmodal interactions. Both tACS behavior and EEG results converged in showing that corticocortical coupling of sensory gamma oscillations is likely involved in processing cross-modal congruence, a fundamental computation in solving the multisensory binding problem. Interestingly, the pattern of results suggests that these sensory interactions should be interpreted in terms of communication rather than feature binding. We propose that the evaluation of cross-modal congruence involves direct crossmodal communication routed by flexible and dynamic coupling and decoupling of sensory gamma oscillations between sensory cortices. As a working hypothesis, we further suggest that phase-coupled gamma oscillations might more generally provide a functional scaffold for cross-modal communication.

\section{References}

Asamoah B, Khatoun A, Mc Laughlin M (2019) Analytical bias accounts for some of the reported effects of tACS on auditory perception. Brain Stimul 12:1001-1009.

Balz J, Romero YR, Keil J, Krebber M, Niedeggen M, Gallinat J, Senkowski1 D (2016) Beta/gamma oscillations and event-related potentials indicate aberrant multisensory processing in schizophrenia. Front Psychol 7:1896.

Bastos AM, Schoffelen JM (2016) A tutorial review of functional connectivity analysis methods and their interpretational pitfalls. Front Syst Neurosci 9:175.

Bolognini N, Frassinetti F, Serino A, Làdavas E (2005) "Acoustical vision" of below threshold stimuli: interaction among spatially converging audiovisual inputs. Exp Brain Res 160:273-282.

Bonnefond M, Jensen O (2015) Gamma activity coupled to alpha phase as a mechanism for top-down controlled gating. PLoS One 10:e0128667.

Botev ZI, Grotowski JF, Kroese DP (2010) Kernel density estimation via diffusion. Ann Stat 38:2916-2957.

Cappe C, Barone P (2005) Heteromodal connections supporting multisensory integration at low levels of cortical processing in the monkey. Eur J Neurosci 22:2886-2902.

Curic S, Leicht G, Thiebes S, Andreou C, Polomac N, Eichler IC, Eichler L, Zöllner C, Gallinat J, Steinmann S, Mulert C (2019) Reduced auditory evoked gamma-band response and schizophrenia-like clinical symptoms under subanesthetic ketamine. Neuropsychopharmacology 44:1239-1246. 
Delorme A, Makeig S (2004) EEGLAB: an open source toolbox for analysis of single-trial EEG dynamics including independent component analysis. J Neurosci Methods 134:9-21.

Desikan RS, Ségonne F, Fischl B, Quinn BT, Dickerson BC, Blacker $D$, Buckner RL, Dale AM, Maguire RP, Hyman BT, Albert MS, Killiany RJ (2006) An automated labeling system for subdividing the human cerebral cortex on MRI scans into gyral based regions of interest. Neuroimage 31:968-980.

Donner TH, Siegel M (2011) A framework for local cortical oscillation patterns. Trends Cogn Sci 15:191-199.

Engel AK, König P, Kreiter AK, Singer W (1991) Interhemispheric synchronization of oscillatory neuronal responses in cat visual cortex. Science 252:1177-1179.

Engel AK, Fries P, Singer W (2001) Dynamic predictions: oscillations and synchrony in top-down processing. Nat Rev Neurosci 2:704716.

Fries $P$ (2009) Neuronal gamma-band synchronization as a fundamental process in cortical computation. Annu Rev Neurosci 32: 209-224.

Fries P (2015) Rhythms for cognition: communication through coherence. Neuron 88:220-235.

Friese U, Daume J, Göschl F, König P, Wang P, Engel AK (2016) Oscillatory brain activity during multisensory attention reflects activation, disinhibition, and cognitive control. Sci Rep 6:32775

Ghazanfar AA, Schroeder CE (2006) Is neocortex essentially multisensory? Trends Cogn Sci 10:278-285.

Gray CM, König P, Engel AK, Singer W (1989) Oscillatory responses in cat visual cortex exhibit inter-columnar synchronization which reflects global stimulus properties. Nature 338:334-337.

Göschl F, Engel AK, Friese U (2014) Attention modulates visualtactile interaction in spatial pattern matching. PLoS One 9:e106896.

Hassler U, Barreto NT, Gruber T (2011) Induced gamma band responses in human EEG after the control of miniature saccadic artifacts. Neuroimage 57:1411-1421.

Helfrich RF, Knepper H, Nolte G, Strüber D, Rach S, Herrmann CS, Schneider TR, Engel AK (2014) Selective modulation of interhemispheric functional connectivity by TACS shapes perception. PLoS Biol 12:e1002031.

Hipp JF, Siegel M (2013) Dissociating neuronal gamma-band activity from cranial and ocular muscle activity in EEG. Front Hum Neurosci 7:338.

Jensen O, Mazaheri A (2010) Shaping functional architecture by oscillatory alpha activity: gating by inhibition. Front Hum Neurosci 4:186.

Kayser C, Petkov Cl, Logothetis NK (2008) Visual modulation of neurons in auditory cortex. Cereb Cortex 18:1560-1574.

Keil J, Senkowski D (2018) Neural oscillations orchestrate multisensory processing. Neuroscientist 24:609-626.

Krebber M, Harwood J, Spitzer B, Keil J, Senkowski D (2015) Visuotactile motion congruence enhances gamma-band activity in visual and somatosensory cortices. Neuroimage 117:160-169.

Liu A, Vöröslakos M, Kronberg G, Henin S, Krause MR, Huang $Y$, Opitz A, Mehta A, Pack CC, Krekelberg B, Berényi A, Parra LC, Melloni L, Devinsky O, Buzsáki G (2018) Immediate neurophysiological effects of transcranial electrical stimulation. Nat Commun 9:5092.

Marshall TR, O'Shea J, Jensen O, Bergmann TO (2015) Frontal eye fields control attentional modulation of alpha and gamma oscillations in contralateral occipitoparietal cortex. J Neurosci 35:16381647.

Misselhorn J, Daume J, Engel AK, Friese U (2016) A matter of attention: crossmodal congruence enhances and impairs performance in a novel trimodal matching paradigm. Neuropsychologia 88:113-122.

Misselhorn J, Friese U, Engel AK (2019) Frontal and parietal alpha oscillations reflect attentional modulation of cross-modal matching. Sci Rep 9:5030.

Mulert C, Leicht G, Hepp P, Kirsch V, Karch S, Pogarell O, Reiser M, Hegerl U, Jäger L, Moller HJ, McCarley RW (2010) Single-trial coupling of the gamma-band response and the corresponding BOLD signal. Neuroimage 49:2238-2247.

Mulert C, Kirsch V, Pascual-Marqui R, McCarley RW, Spencer KM (2011) Long-range synchrony of $\gamma$ oscillations and auditory hallucination symptoms in schizophrenia. Int J Psychophysiol 79:5563.

Nolte G, Dassios G (2005) Analytic expansion of the EEG lead field for realistic volume conductors. Phys Med Biol 50:3807-3823.

Nolte G, Bai O, Wheaton L, Mari Z, Vorbach S, Hallett M (2004) Identifying true brain interaction from EEG data using the imaginary part of coherency. Clin Neurophysiol 115:2292-2307.

Noury N, Siegel M (2017) Phase properties of transcranial electrical stimulation artifacts in electrophysiological recordings. Neuroimage 158:406-416.

Noury N, Hipp JF, Siegel M (2016) Physiological processes nonlinearly affect electrophysiological recordings during transcranial electric stimulation. Neuroimage 140:99-109.

Pascual-Marqui RD, Lehmann D, Koukkou M, Kochi K, Anderer P, Saletu B, Tanaka H, Hirata K, John ER, Prichep L, Biscay-Lirio R, Kinoshita T (2011) Assessing interactions in the brain with exact low-resolution electromagnetic tomography. Philos Transact A Math Phys Eng Sci 369:3768-3784.

Patel J, Bansal V, Minha P, Ho J, Datta A, Bikson M (2009) Highdensity transcranial direct current stimulation (HD-tDCS): skin safety and comfort. J Med Devices 3:027554.

Polanía R, Nitsche MA, Korman C, Batsikadze G, Paulus W (2012) The importance of timing in segregated theta phase-coupling for cognitive performance. Curr Biol 22:1314-1318.

Raij T, Ahveninen J, Lin FH, Witzel T, Jääskeläinen IP, Letham B, Israeli E, Sahyoun C, Vasios C, Stufflebeam S, Hämäläinen M, Belliveau JW (2010) Onset timing of cross-sensory activations and multisensory interactions in auditory and visual sensory cortices. Eur J Neurosci 31:1772-1782.

Riecke L, Sack AT, Schroeder CE (2015) Endogenous delta/theta sound-brain phase entrainment accelerates the buildup of auditory streaming. Curr Biol 25:3196-201.

Romei V, Brodbeck V, Michel C, Amedi A, Pascual-Leone A, Thut G (2008) Spontaneous fluctuations in posterior $\alpha$-band EEG activity reflect variability in excitability of human visual areas. Cereb Cortex 18:2010-2018

Saturnino GB, Antunes A, Thielscher A (2015) On the importance of electrode parameters for shaping electric field patterns generated by tDCS. Neuroimage 120:25-35.

Saturnino GB, Madsen KH, Siebner HR, Thielscher A (2017) How to target inter-regional phase synchronization with dual-site transcranial alternating current stimulation. Neuroimage 163:68-80.

Scheeringa R, Fries P, Petersson KM, Oostenveld R, Grothe I, Norris DG, Hagoort P, Bastiaansen MC (2011) Neuronal dynamics underlying high-and low-frequency EEG oscillations contribute independently to the human BOLD signal. Neuron 69:572-583.

Schneider TR, Debener S, Oostenveld R, Engel AK (2008a) Enhanced EEG gamma-band activity reflects multisensory semantic matching in visual-to-auditory object priming. Neuroimage 42: $1244-1254$

Schneider TR, Engel AK, Debener S (2008b) Multisensory identification of natural objects in a two-way crossmodal priming paradigm. Exp Psychol 55:121-132.

Schwab BC, Misselhorn J, Engel AK (2019) Modulation of large-scale cortical coupling by transcranial alternating current stimulation. Brain Stimul 12:1187-1196.

Senkowski D, Talsma D, Herrmann CS, Woldorff MG (2005) Multisensory processing and oscillatory gamma responses: effects of spatial selective attention. Exp Brain Res 166:411-426.

Senkowski D, Talsma D, Grigutsch M, Herrmann CS, Woldorff MG (2007) Good times for multisensory integration: effects of the precision of temporal synchrony as revealed by gamma-band oscillations. Neuropsychologia 45:561-571.

Senkowski D, Schneider TR, Foxe J, Engel AK (2008) Crossmodal binding through neural coherence: implications for multisensory processing. Trends Neurosci 31:301-409. 
Spence C (2011) Crossmodal correspondences: a tutorial review. Atten Percept Psychophys 73:971-995.

Stone DB, Coffman BA, Bustillo JR, Aine CJ, Stephen JM (2014) Multisensory stimuli elicit altered oscillatory brain responses at gamma frequencies in patients with schizophrenia. Front Hum Neurosci 8:788.

Tallon-Baudry C, Bertrand O, Delpuech C, Pernier J (1996) Stimulus specificity of phase-locked and non-phase-locked $40 \mathrm{~Hz}$ visual responses in human. J Neurosci 16:4240-4249.

Treisman A (1996) The binding problem. Curr Opin Neurobiol 6:171-178.

Uhlhaas PJ, Singer W (2010) Abnormal neural oscillations and synchrony in schizophrenia. Nat Rev Neurosci 11:100-113. van Es MWJ, Schoffelen JM (2019) Stimulus-induced gamma power predicts the amplitude of the subsequent visual evoked response. Neuroimage 186:703-712.

Watson AB, Pelli DG (1983) QUEST: a Bayesian adaptive psychometric method. Percept Psychophys 33:113-120.

Wöstmann M, Vosskuhl J, Obleser J, Herrmann CS (2018) Opposite effects of lateralised transcranial alpha versus gamma stimulation on auditory spatial attention. Brain Stimul 11:752-758.

Yuval-Greenberg S, Deouell LY (2007) What you see is not (always) what you hear: induced gamma band responses reflect crossmodal interactions in familiar object recognition. J Neurosci 27: 1090-1096. 